\title{
Publisher Correction: Indole 3-acetic acid, indoxyl sulfate and paracresyl-sulfate do not influence anemia parameters in hemodialysis patients
}

BMC Nephrology

\section{Erratum}

In the original publication of this article [1], Table 3 is incorrect, several cell values in column 4 (no ESA $(n=8))$ have been incorrectly shifted during typesetting.

In this Erratum the correct and incorrect version of Table 3 are published.

The publisher apologizes for the inconvenience caused by this error to the authors and readers.

Received: 18 September 2017 Accepted: 18 September 2017

Published online: 04 October 2017

\section{Reference}

1. Bataille, et al. Indole 3-acetic acid, indoxyl sulfate and paracresyl-sulfate do not influence anemia parameters in hemodialysis patients. BMC Nephrol. 2017;18:251. doi: 10.1186/s12882-017-0668-5.

\footnotetext{
* Correspondence: info@biomedcentral.com
} London, UK 
Table 1 Incorrect version of Table 3 as published on 26 July 2017. The incorrect cells are displayed in italics

\begin{tabular}{|c|c|c|c|c|c|c|c|}
\hline & \multicolumn{3}{|l|}{$\mathrm{Hb}>10 \mathrm{~g} / \mathrm{dL}$} & \multicolumn{3}{|l|}{$\mathrm{Hb} \leq 10 \mathrm{~g} / \mathrm{dL}$} & \multirow[t]{2}{*}{$p$-value } \\
\hline & $\begin{array}{l}\text { no ESA } \\
(n=63)\end{array}$ & $\begin{array}{l}\mathrm{ESA} \leq 40 \mu \mathrm{g} / \mathrm{w} \\
(n=75)\end{array}$ & $\begin{array}{l}\mathrm{ESA}>40 \mu \mathrm{g} / \mathrm{w} \\
(n=41)\end{array}$ & $\begin{array}{l}\text { no ESA } \\
(n=8)\end{array}$ & $\begin{array}{l}\mathrm{ESA} \leq 40 \mu \mathrm{g} / \mathrm{w} \\
(n=25)\end{array}$ & $\begin{array}{l}\mathrm{ESA}>40 \mu \mathrm{g} / \mathrm{w} \\
(n=28)\end{array}$ & \\
\hline Age (years) & $67.8 \pm 15.3$ & $67.1 \pm 16.2$ & $69.9 \pm 15.4$ & $74.5 \pm 8.1$ & $64.8 \pm 17.6$ & $65.9 \pm 18.3$ & 0.63 \\
\hline Gender (male) & $61.9 \%$ & $58.7 \%$ & $41.5 \%$ & $62.5 \%$ & $64.0 \%$ & $42.9 \%$ & 0.19 \\
\hline Diabetes & $44.4 \%$ & $41.3 \%$ & $43.9 \%$ & $37.5 \%$ & $48.0 \%$ & $35.7 \%$ & 0.96 \\
\hline Hemoglobin (g/dL) & $11.6 \pm 0.9$ & $11.2 \pm 0.8$ & $11.1 \pm 0.8$ & $9.6 \pm 0.6$ & $9.5 \pm 0.4$ & $9.0 \pm 0.9$ & $<0.001$ \\
\hline TSAT (\%) & $29.2 \pm 13.5$ & $23.5 \pm 9.2$ & $22.7 \pm 11.1$ & $45.8 \pm 27.4$ & $25.6 \pm 9.8$ & $20.1 \pm 17.4$ & $<0.001$ \\
\hline Ferritin $(\mathrm{ng} / \mathrm{mL})$ & $480 \pm 330$ & $426 \pm 285$ & $473 \pm 304$ & $1341 \pm 1541$ & $636 \pm 314$ & $677 \pm 901$ & $<0.001$ \\
\hline Albumin (g/L) & $38.4 \pm 4.4$ & $39.2 \pm 4.1$ & $37.5 \pm 4.8$ & & $37.8 \pm 4.6$ & $33.0 \pm 7.8$ & $<0.001$ \\
\hline$\beta 2$ microglobulin (mg/L) & $26.2 \pm 7.0$ & $25.7 \pm 6.9$ & $27.4 \pm 8.2$ & $34.1 \pm 6.4$ & $25.6 \pm 6.2$ & $27.4 \pm 7.0$ & 0.008 \\
\hline Parathormone $(\mathrm{pg} / \mathrm{mL})$ & $268 \pm 318$ & $284 \pm 315$ & $195 \pm 207$ & $35.8 \pm 9.8$ & $264 \pm 354$ & $237 \pm 438$ & 0.83 \\
\hline $\begin{array}{l}\text { Predialysis creatinine } \\
(\mu \mathrm{mol} / \mathrm{L})\end{array}$ & $764 \pm 282$ & $769 \pm 242$ & $684 \pm 250$ & $224 \pm 299$ & $757 \pm 220$ & $638 \pm 239$ & 0.08 \\
\hline Predialysis urea (mmol/L) & $20.8 \pm 5.9$ & $21.7 \pm 6.7$ & $20.0 \pm 6.5$ & $623 \pm 153$ & $21.0 \pm 6.1$ & $19.6 \pm 8.3$ & 0.39 \\
\hline C-reactive protein (mg/L) & $12.2 \pm 18.9$ & $12.7 \pm 28.4$ & $26.0 \pm 67.4$ & $17.2 \pm 5.5$ & $23.1 \pm 49.9$ & $36.5 \pm 37.1$ & 0.02 \\
\hline IAA $(\mu \mathrm{mol} / \mathrm{L})$ & $5.10 \pm 5.28$ & $5.73 \pm 5.97$ & $3.51 \pm 3.50$ & $51.9 \pm 97.3$ & $4.67 \pm 3.05$ & $3.78 \pm 2.60$ & 0.09 \\
\hline IS ( $\mu \mathrm{mol} / \mathrm{L})$ & $92.8 \pm 54.7$ & $97.9 \pm 45.2$ & $100.9 \pm 49.9$ & $2.29 \pm 1.30$ & $85.2 \pm 55.5$ & $82.3 \pm 61.9$ & 0.54 \\
\hline $\mathrm{PCS}(\mu \mathrm{mol} / \mathrm{L})$ & $169 \pm 101$ & $172 \pm 94$ & $150 \pm 97$ & $78.1 \pm 37.6$ & $175 \pm 98$ & $96 \pm 99$ & 0.01 \\
\hline \multicolumn{8}{|l|}{ Iron medication } \\
\hline IV iron medication & $54.0 \%$ & $68.0 \%$ & $73.2 \%$ & $135 \pm 75$ & $48.0 \%$ & $64.3 \%$ & 0.10 \\
\hline Iron dose (mg/mo) & $175 \pm 188$ & $228 \pm 245$ & $351 \pm 318$ & $163 \pm 292$ & $124 \pm 167$ & $314 \pm 374$ & 0.002 \\
\hline HDF (\%) & $54.0 \%$ & $30.7 \%$ & $31.7 \%$ & $50.0 \%$ & $52.0 \%$ & $46.4 \%$ & 0.05 \\
\hline $\mathrm{Kt} / \mathrm{N}$ & $1.51 \pm 0.26$ & $1.50 \pm 0.28$ & $1.50 \pm 0.42$ & $1.46 \pm 0.30$ & $1.57 \pm 0.26$ & $1.51 \pm 0.34$ & 0.95 \\
\hline
\end{tabular}


Table 2 Correct version of Table 3, the corrected cells are displayed in bold

\begin{tabular}{|c|c|c|c|c|c|c|c|}
\hline & \multicolumn{3}{|c|}{$\mathrm{Hb}>10 \mathrm{~g} / \mathrm{dL}$} & \multicolumn{3}{|l|}{$\mathrm{Hb} \leq 10 \mathrm{~g} / \mathrm{dL}$} & \multirow[t]{2}{*}{$p$-value } \\
\hline & $\begin{array}{l}\text { no ESA } \\
(n=63)\end{array}$ & $\begin{array}{l}\text { ESA } \leq 40 \mu \mathrm{g} / \mathrm{w} \\
(n=75)\end{array}$ & $\begin{array}{l}\mathrm{ESA}>40 \mu \mathrm{g} / \mathrm{w} \\
(n=41)\end{array}$ & $\begin{array}{l}\text { no ESA } \\
(n=8)\end{array}$ & $\begin{array}{l}\mathrm{ESA} \leq 40 \mu \mathrm{g} / \mathrm{w} \\
(n=25)\end{array}$ & $\begin{array}{l}\mathrm{ESA}>40 \mu \mathrm{g} / \mathrm{w} \\
(n=28)\end{array}$ & \\
\hline Age (years) & $67.8 \pm 15.3$ & $67.1 \pm 16.2$ & $69.9 \pm 15.4$ & $74.5 \pm 8.1$ & $64.8 \pm 17.6$ & $65.9 \pm 18.3$ & 0.63 \\
\hline Gender (male) & $61.9 \%$ & $58.7 \%$ & $41.5 \%$ & $62.5 \%$ & $64.0 \%$ & $42.9 \%$ & 0.19 \\
\hline Diabetes & $44.4 \%$ & $41.3 \%$ & $43.9 \%$ & $37.5 \%$ & $48.0 \%$ & $35.7 \%$ & 0.96 \\
\hline Hemoglobin (g/dL) & $11.6 \pm 0.9$ & $11.2 \pm 0.8$ & $11.1 \pm 0.8$ & $9.6 \pm 0.6$ & $9.5 \pm 0.4$ & $9.0 \pm 0.9$ & $<0.001$ \\
\hline TSAT (\%) & $29.2 \pm 13.5$ & $23.5 \pm 9.2$ & $22.7 \pm 11.1$ & $45.8 \pm 27.4$ & $25.6 \pm 9.8$ & $20.1 \pm 17.4$ & $<0.001$ \\
\hline Ferritin $(\mathrm{ng} / \mathrm{mL})$ & $480 \pm 330$ & $426 \pm 285$ & $473 \pm 304$ & $1341 \pm 1541$ & $636 \pm 314$ & $677 \pm 901$ & $<0.001$ \\
\hline Albumin (g/L) & $38.4 \pm 4.4$ & $39.2 \pm 4.1$ & $37.5 \pm 4.8$ & $34.1 \pm 6.4$ & $37.8 \pm 4.6$ & $33.0 \pm 7.8$ & $<0.001$ \\
\hline ß2 microglobulin (mg/L) & $26.2 \pm 7.0$ & $25.7 \pm 6.9$ & $27.4 \pm 8.2$ & $35.8 \pm 9.8$ & $25.6 \pm 6.2$ & $27.4 \pm 7.0$ & 0.008 \\
\hline Parathormone $(\mathrm{pg} / \mathrm{mL})$ & $268 \pm 318$ & $284 \pm 315$ & $195 \pm 207$ & $224 \pm 299$ & $264 \pm 354$ & $237 \pm 438$ & 0.83 \\
\hline $\begin{array}{l}\text { Predialysis creatinine } \\
(\mu \mathrm{mol} / \mathrm{L})\end{array}$ & $764 \pm 282$ & $769 \pm 242$ & $684 \pm 250$ & $623 \pm 153$ & $757 \pm 220$ & $638 \pm 239$ & 0.08 \\
\hline Predialysis urea (mmol/L) & $20.8 \pm 5.9$ & $21.7 \pm 6.7$ & $20.0 \pm 6.5$ & $17.2 \pm 5.5$ & $21.0 \pm 6.1$ & $19.6 \pm 8.3$ & 0.39 \\
\hline $\begin{array}{l}\text { C-reactive protein (mg/ } \\
\text { L) }\end{array}$ & $12.2 \pm 18.9$ & $12.7 \pm 28.4$ & $26.0 \pm 67.4$ & $51.9 \pm 97.3$ & $23.1 \pm 49.9$ & $36.5 \pm 37.1$ & 0.02 \\
\hline IAA $(\mu \mathrm{mol} / \mathrm{L})$ & $5.10 \pm 5.28$ & $5.73 \pm 5.97$ & $3.51 \pm 3.50$ & $2.29 \pm 1.30$ & $4.67 \pm 3.05$ & $3.78 \pm 2.60$ & 0.09 \\
\hline IS ( $\mu \mathrm{mol} / \mathrm{L})$ & $92.8 \pm 54.7$ & $97.9 \pm 45.2$ & $100.9 \pm 49.9$ & $78.1 \pm 37.6$ & $85.2 \pm 55.5$ & $82.3 \pm 61.9$ & 0.54 \\
\hline $\mathrm{PCS}(\mu \mathrm{mol} / \mathrm{L})$ & $169 \pm 101$ & $172 \pm 94$ & $150 \pm 97$ & $135 \pm 75$ & $175 \pm 98$ & $96 \pm 99$ & 0.01 \\
\hline \multicolumn{8}{|l|}{ Iron medication } \\
\hline IV iron medication & $54.0 \%$ & $68.0 \%$ & $73.2 \%$ & $37.5 \%$ & $48.0 \%$ & $64.3 \%$ & 0.10 \\
\hline Iron dose (mg/mo) & $175 \pm 188$ & $228 \pm 245$ & $351 \pm 318$ & $163 \pm 292$ & $124 \pm 167$ & $314 \pm 374$ & 0.002 \\
\hline HDF $(\%)$ & $54.0 \%$ & $30.7 \%$ & $31.7 \%$ & $50.0 \%$ & $52.0 \%$ & $46.4 \%$ & 0.05 \\
\hline $\mathrm{Kt} / \mathrm{N}$ & $1.51 \pm 0.26$ & $1.50 \pm 0.28$ & $1.50 \pm 0.42$ & $1.46 \pm 0.30$ & $1.57 \pm 0.26$ & $1.51 \pm 0.34$ & 0.95 \\
\hline
\end{tabular}

